\title{
Microscopic Theory of the Nuclear Equation of State and Neutron Star Structure
}

\author{
Marcello Baldo and Fiorella Burgio \\ Istituto Nazionale di Fisica Nucleare, Sez. Catania, and Universitá di Catania, \\ Corso Italia 57, 95129 Catania, Italy
}

\begin{abstract}
The Bethe-Brueckner-Goldstone many-body theory of the Nuclear Equation of State is reviewed in some details. In the theory, one performs an expansion in terms of the Brueckner two-body scattering matrix and an ordering of the corresponding many-body diagrams according to the number of their hole-lines. Recent results are reported, both for symmetric and for pure neutron matter, based on realistic twonucleon interactions. It is shown that there is strong evidence of convergence in the expansion. Once three-body forces are introduced, the phenomenological saturation point is reproduced and the theory is applied to the study of neutron star properties. One finds that in the interior of neutron stars the onset of hyperons strongly softens the Nuclear Equation of State. As a consequence, the maximum mass of neutron stars turns out to be at the lower limit of the present phenomenological observation.
\end{abstract}

\section{Introduction}

It is believed that macroscopic portions of (asymmetric) nuclear matter form the interior bulk part of neutron stars, commonly associated with pulsars. Despite infinite nuclear matter is obviously an idealized physical system, the theoretical determination of the corresponding Equation of State is, therefore, an essential step towards the understanding of the physical properties of neutron stars. On the other hand, the comparison of the theoretical predictions on neutron stars with the experimental observations can provide serious constraints on the $\mathrm{Nu}$ clear Equation of State. Unfortunately, neutron stars are elusive astrophysical objects, and only indirect observations of their structure, including their sizes and masses, are possible. However, the astrophysics of neutron stars is rapidly developing, in view of the observations coming from the new generation of artificial satellites, and one can expect that it will be possible in the near future to confront the theoretical predictions with more and more stringent phenomenological data.

Heavy ion reactions is another field of research where the nuclear Equation of State (EOS) is a relevant issue. In this case, the difficulty of extracting the EOS is due to the complexity of the processes, since the interpretation of the data is necessarily linked to the analysis of the reaction mechanism. An enormous amount of work has been done in the last two decades in the field, but clear indications about the main characteristics of the EOS have still to come. Furthermore, the typical time scale of heavy ion reactions is enormously different from the typical neutron star time scale, and this can prevent a direct link between the two field of research. In particular, nuclear matter inside neutron stars 
is completely catalized, namely it is quite close to the ground state, reachable also by weak processes. In heavy ion reactions the evolution is too rapid to allow weak processes to relax the system towards such a catalized state, and therefore the tested Equation of State can differ from the neutron star one, especially at high density.

On the theoretical side, the main general difficulty is the treatment of the strong repulsive core, which dominates the short range behaviour of the nucleonnucleon $(\mathrm{NN})$ interaction, typical of the nuclear system, but which is common to other systems like liquid helium. Simple perturbation theory cannot of course be applied, since the matrix elements of the interaction are too large. One way of overcoming this difficulty is to introduce the two-body scattering G-matrix, which has a much smoother behaviour even for strong repulsive core. It is possible to rearrange the perturbation expansion in terms of the reaction G-matrix, in place of the original bare $\mathrm{NN}$ interaction, and this procedure is systematically exploited in the Bethe-Brueckner-Goldstone (BBG) expansion [1]. In this contribution we present the latest results on the nuclear EOS based on BBG expansion and their applications to the physics of neutron stars.

\section{The BBG expansion and the nuclear EOS}

The BBG expansion for the ground state energy at a given density, i.e. the EOS at zero temperature, can be ordered according to the number of independent hole-lines appearing in the diagrams representing the different terms of the expansion. This grouping of diagrams generates the so-called hole-line expansion [2]. The smallness parameter of the expansion is assumed to be the "wound parameter" [2], roughly determined by the ratio between the core volume and the volume per particle in the system. It gives an estimate of the decreasing factor introduced by an additional hole-line in the diagram series. The parameter turns out to be small enough up to 2-3 times nuclear matter saturation density. The diagrams with a given number $n$ of hole-lines are assumed to describe the main contribution to the $n$-particle correlations in the system. At the two hole-line level of approximation the corresponding summation of diagrams produces the Brueckner-Hartree-Fock (BHF) approximation, which incorporates the two particle correlations. The BHF approximation includes the self-consistent procedure of determining the single particle auxiliary potential, which is an essential ingredient of the method. Once the auxiliary self-consistent potential is introduced, the expansion is implemented by introducing the set of diagrams which include "potential insertions". To be specific, the introduction of the auxiliary potential can be formally performed by splitting the hamiltonian in a modified way from the usual one

$$
H=T+V=T+U+(V-U) \equiv H_{0}^{\prime}+V^{\prime}
$$

where $T$ is the kinetic energy and $V$ the nucleon-nucleon interaction. Then one consider $V^{\prime}=V-U$ as the new interaction potential and $H_{0}^{\prime}$ as the new single 
particle hamiltonian. Then, the single particle energy $e(k)$ is given by

$$
e(k)=\frac{\hbar^{2} k^{2}}{2 m}+U(k)
$$

while $U$ must be chosen in such a way that the new interaction $V^{\prime}$ is, in some sense, "reduced" with respect to the original one $V$, so that the expansion in $V^{\prime}$ should be faster converging. The introduction of the auxiliary potential turns out to be essential, otherwise the hole-expansion would be badly diverging. The total energy $E$ can then be written as

$$
E=\sum_{k} e(k)+B
$$

where $B$ is the interaction energy due to $V^{\prime}$.

The BHF sums the so called "ladder diagrams". Some of them are depicted in Fig. 1. One has to consider this set of diagrams where one, two, three, and so one, two-body interactions $v$ appear, including exchange terms. Special care must be used in counting correctly the diagrams which give the same contribution.

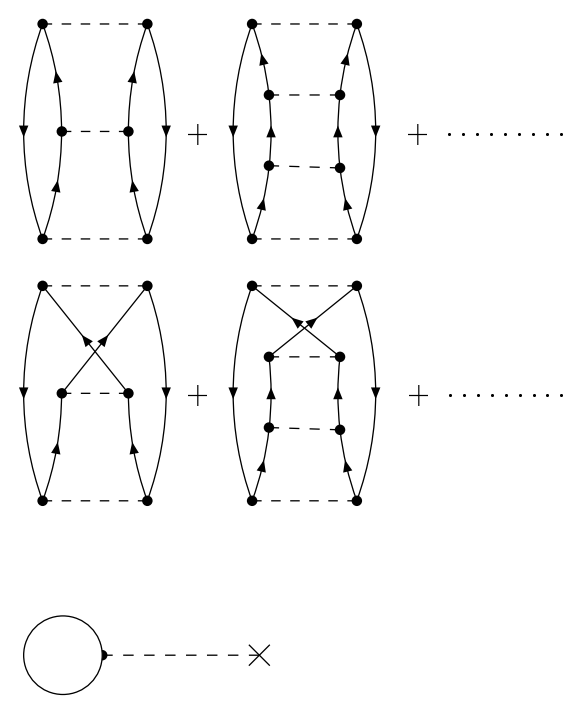

Fig. 1. Third and forth order ladder diagrams in the bare interaction (dashed lines) and first order potential insertion (bottom).

The repeated action of the two-body potential $v$ clearly describes the scattering of two nucleons which lie above the Fermi sphere. The summation of the ladder 
diagrams can be performed by solving the integral equation for the Brueckner G-matrix

$$
\begin{gathered}
\left\langle k_{1} k_{2}|G(\omega)| k_{3} k_{4}\right\rangle=\left\langle k_{1} k_{2}|v| k_{3} k_{4}\right\rangle+ \\
+\sum_{k_{3}^{\prime} k_{4}^{\prime}}\left\langle k_{1} k_{2}|v| k_{3}^{\prime} k_{4}^{\prime}\right\rangle \frac{\left(1-\Theta_{F}\left(k_{3}^{\prime}\right)\right)\left(1-\Theta_{F}\left(k_{4}^{\prime}\right)\right)}{\omega-e_{k_{3}^{\prime}}-e_{k_{4}^{\prime}}}\left\langle k_{3}^{\prime} k_{4}^{\prime}|G(\omega)| k_{3} k_{4}\right\rangle
\end{gathered}
$$

where $\Theta_{F}(k)=1$ for $k<k_{F}$ and is zero otherwise, being $k_{F}$ the Fermi momentum. The product $Q\left(k, k^{\prime}\right)=\left(1-\Theta_{F}(k)\right)\left(1-\Theta_{F}\left(k^{\prime}\right)\right)$, appearing in the kernel of Eq. (4), enforces the scattered momenta to lie outside the Fermi sphere, and it is commonly referred as the "Pauli operator". This G-matrix can be viewed as the in-medium scattering matrix between two nucleons. It has to be stressed that the scattering G-matrix depends parametrically on the entry energy $\omega$, namely it is defined in general also off-shell, as the usual scattering matrix in vacuum. The self-consistent single particle potential $U(k)$ is determined by the equation

$$
U(k)=\sum_{k^{\prime}<k_{F}}\left\langle k k^{\prime}\left|G\left(e_{k_{1}}+e_{k_{2}}\right)\right| k k^{\prime}\right\rangle_{A}
$$

with $\left|k k^{\prime}\right\rangle_{A}=\left|k k^{\prime}\right\rangle-\left|k k^{\prime}\right\rangle$.

According to the definition of Eq. (2), Eq. (5) implies an implicit self-consistent procedure.

Summing up the ladder diagrams to all orders, one then gets the two diagrams, direct and exchange, of Fig. 2, where a wavy lines indicates a Brueckner G-matrix. Indeed, if one expands the G-matrix from Eq. (4), in terms of the bare interaction $v$, and inserts the expansion in the diagrams of Fig. 2, one gets the full sets of ladder diagrams, indicated in Fig. 1. More details on the rules for writing down the explicit expression of the diagrams can be found in ref. [1].
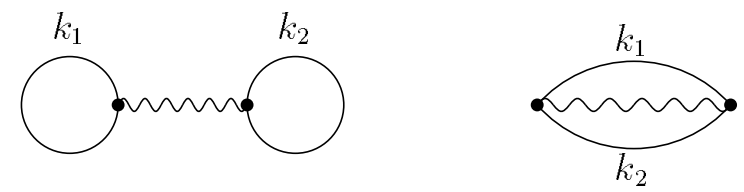

Fig. 2. The two hole-line contribution in terms of the Brueckner G-matrix (wavy line).

The first potential insertion diagram, at the bottom of Fig. 1, cancels out the potential part of the single particle energy of Eq. (2), in the expression for the total energy $E$. This is actually true for any definition of the auxiliary potential $U$. At the two hole-line level of approximation, one therefore gets

$$
\begin{aligned}
E & =\sum_{k<k_{F}} \frac{\hbar^{2} k^{2}}{2 m}+\frac{1}{2} \sum_{k, k^{\prime}<k_{F}}\left\langle k k^{\prime}\left|G\left(e_{k}+e_{k^{\prime}}\right)\right| k k^{\prime}\right\rangle_{A} \\
& \equiv \sum_{k<k_{F}} \frac{\hbar^{2} k^{2}}{2 m}+\frac{1}{2} \sum_{k<k_{F}} U(k)
\end{aligned}
$$


where, in the last equality, the definition of Eq. (5) has been adopted. The result that only the unperturbed kinetic energy appears in the expression for $E$, and all the correlations are included in the potential energy part, holds true to all orders and it is a peculiarity of the BBG expansion. Of course, the modification of the momentum distribution, and therefore of the kinetic energy, is included in the interaction energy part, but it is treated on the same footing as the other correlation effects. This seems to present a noticeable advantage. In fact, the modification of the kinetic energy in itself is quite large and, of course, positive and should be therefore compensate by an extremely accurate calculations of the (negative) correlation energy. On the other hand, putting the two effects on the same footing, one can expect that strong cancellation occur order by order.

Let us now discuss the choice of the single particle potential $U$. As it was discussed in connection with Eq. (11), the potential $U$ is in principle arbitrary, and it is used only as a tool for speed up the convergence of the expansion. However, physical considerations suggest the self-consistent procedure defined by Eq. (5) to obtain the potential $U$. The self-consistency condition is clearly non-perturbative and it is a generalization of the usual Hartree-Fock (HF) approximation, namely the Brueckner G-matrix is used in place of the bare NN interaction $v$. For nuclear matter the HF approximation would produce unrealistic results, because of the strong repulsive core. The G-matrix takes into account the short range correlations between pairs of nucleons, and therefore it gives a much improved balance between attractive and repulsive contributions. The approximation of Eq. (6), together with Eqs. (2), (5), is usually referred to as the Brueckner-Hartree-Fock (BHF) approximation. This definition of $U$ corresponds to the diagrams of Fig. 3 .
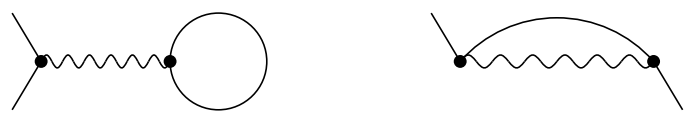

Fig. 3. The direct and exchange parts of the auxiliary potential $U$ in terms of the Brueckner G-matrix.

It has to be noticed that the G-matrix appearing in the diagrams are calculated on-shell, according to Eq. (阿), i.e. its entry energy is equal to the energy of the two particles with the two entry momenta. Therefore the total energy at the BHF level of approximation can be written also in terms of the potential $U$, as in the second line of Eq. (6).

In the general BBG expansion, in all the higher order diagrams, beyond the BHF approximation, the same definition of $U$ is kept and the bare NN potential is replaced by the G-matrix by performing the corresponding ladder sums whenever it is possible. In this way the diagrammatic expansion is rearranged in terms of 
the Brueckner G-matrix, in place of the bare NN interaction, with the only obvious prescription that no ladder sums can now appear in the diagrams, just to avoid double counting.

We have seen that the ladder sum at the BHF level introduce on-shell Gmatrices only. This is not necessarily the case if the ladder sum is performed inside a generic higher order energy diagram, since then the entry energy of the resulting G-matrix depends in general on the rest of the diagrams. The energy denominators appearing in the BBG expansion include, in fact, all the particle and hole energies across the diagram. This point will be discuss later and we will see that some exceptions to this expectation can occur.

Another strong reason in favour of keeping the BHF definition for the single particle potential $U$ in the general BBG expansion is the occurrence of cancellation between diagrams including three hole-lines, thus reducing the relevance of higher order contributions. This is true for the two diagrams reported in Fig. 1 . The diagram $(b)$ in the right side of the figure is a potential insertion diagram, where the dashed line with the cross indicates a multiplication by a factor $U(k)$, being $k$ the momentum of the hole-line to which the potential is attached. The rule for writing down the potential insertion diagrams can also be found in ref. [1]. The diagram $(a)$ in the left side of Fig. 目 contains a G-matrix loop in place of the potential $U$. If the G-matrix is on-shell,in view of the definition of Eq. (5) and the graphical rules, one can easily see that the two diagrams cancel out exactly.

( a )

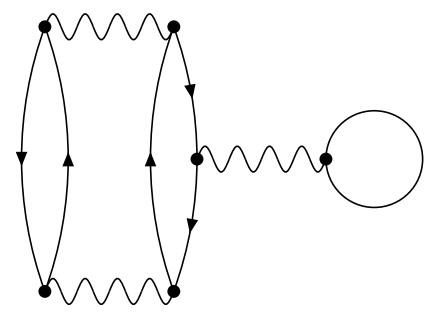

( b )

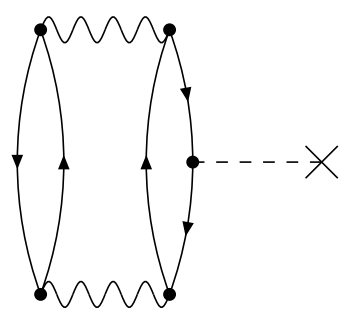

Fig. 4. Lowest order three hole-line diagram (a) and the corresponding potential insertion diagram (b).

At first site the G-matrix of diagram ( $a$ ) should be not calculated on-shell. However, it has been shown in ref. [3] that, if the ladder sums included in the diagram contain bare interactions which appear in all possible position along the diagram, then their overall contribution reduces indeed to the diagram $(a)$ of Fig. 4 , with the G-matrix calculated on-shell, and the above mentioned cancellation holds true.

The definition of Eq. (5) does not specify completely the single particle potential $U(k)$. For momenta $k>k_{F}$ the value of the potential $U(k)$ does not 
appear explicitly in the energy expression of Eq. (6) at the BHF level. In old BHF calculations the potential $U(k)$ was then taken identically zero above the Fermi momentum, with the justification that the interaction between particles above $k_{F}$ is expected to be small and anyhow only slightly affecting the total energy. In this choice, usually referred to as "standard choice", the potential has then a jump at $k_{F}$. For this reason it is also often called "gap choice". Most modern BHF calculations adopt a potential $U(k)$ which is defined by extending the definition of Eq. (5) also above $k_{F}$, thus making $U(k)$ continuous across the Fermi sphere. This definition modifies the self-consistent equation and therefore also the potential for $k<k_{F}$. As a consequence, this different choice, usually called "continuous choice", modifies indirectly also the value of the BHF energy of Eq. (6). There are some arguments in favour of the continuous choice. Since $U(k)$ has the physical meaning of single particle potential, it is intimately related to the single particle self-energy. Indeed, one can show that $U(k)$ is the onshell self energy to first order in the hole expansion. As such, the potential $U(k)$ must be a continuous function of the momentum. Another point to be considered is related to the two other three hole-line diagrams depicted in Fig. 5. They can be obtained from the diagrams of Fig. 1 just by attaching the intermediate Gmatrix (diagram $a$ ) and the potential $U$ (diagram $b$ ) to the particle-line instead of the hole-line. Diagram $(a)$ is usually denoted as "bubble diagram". In this case the G-matrix is not calculated on-shell, since the argumentation of ref. [3] does not apply, and no exact cancellation can occur between the two diagrams. Actually, in the standard choice the potential insertion diagram $b$ is identically zero, since in this case $U(k)$ vanishes for $k>k_{F}$. On the contrary, in the continuous choice, the potential insertion diagram does not vanish, and some degree of cancellation can be expected, despite the G-matrix is calculated off-shell, thus reducing also in this case the contribution from higher order diagrams.

( a )

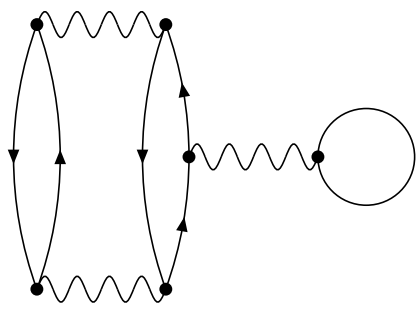

( b )

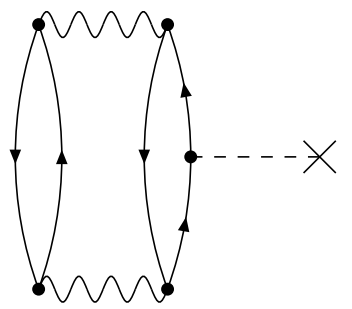

Fig. 5. Bubble three hole-line diagram (a) and the corresponding potential insertion diagram (b)

At first sight it can be surprising that the final result for the nuclear matter EOS could depend on the choice of the single particle potential $U$, since the 
splitting of Eq. (11) is a trivial identity and the final result should be independent on the particular choice of $U(k)$. This is of course true only if the full BBG expansion to all order could be summed up exactly. If the expansion is truncated at a given order, the results can show still a dependence on the choice of $U(k)$, and this dependence will be stronger more the expansion is far from a reasonable convergence. One can, therefore, take the degree of the dependence on $U(k)$ as a measure of the degree of convergence reached at a given order of the expansion. The gap and continuous choices can be considered two opposite cases for the potential $U(k)$, since any other reasonable choice would modify mainly its definition for $k>k_{F}$ and would be somehow intermediate between these two

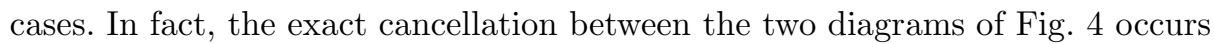
only with the definition of Eq. (5) for $k<k_{F}$, and it appears inconvenient to adopt a choice for $U(k)$ which does not include the cancellation. However, other choices are surely possible, and one should check also in those other cases the degree of convergence reached at a given level of the expansion. In the sequel we will restrict to the gap and continuous choices for checking the convergence of the expansion.

Let us consider the symmetric nuclear matter EOS at the BHF level of approximation. The results for the two choices for $U(k)$ are reported in Fig. 6, where the Argonne $\mathrm{v}_{14}[5]$ is used for the bare NN potential.

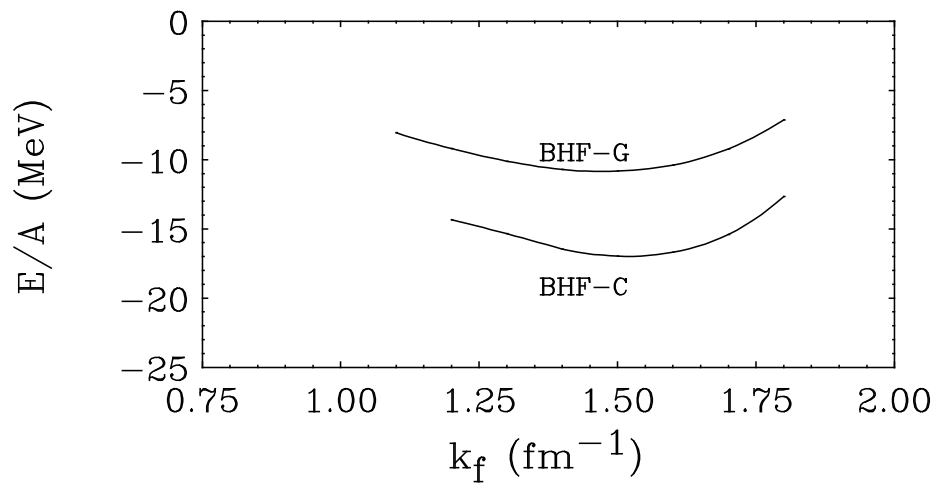

Fig. 6. Nuclear matter saturation curve for the Argonne $\mathrm{v}_{14}$ NN potential. The solid lines indicate the results at the Brueckner (two hole-lines) level for the standard (BHF$\mathrm{G})$ and the continuous choices (BHF-C) respectively.

It is apparent from the figure that the degree of convergence is not yet satisfactory at the BHF level. The difference for the energy per particle is of about 4-5 $\mathrm{MeV}$ in the considered density range. It has to be kept in mind, however, that the potential energy part of the binding energy of Eq. 6 is about $-40 \mathrm{MeV}$ around saturation density, and therefore the discrepancy between the two choices 
is of about $10 \%$. This is the expected degree of convergence at the BHF level, according to the above discussed criterion.

The BHF results imply that, for a check of convergence, it is mandatory to consider the three hole-line diagrams contribution. According to the BBG expansion, this set of diagrams describes the irreducible three-nucleon correlations, i.e. the three-body correlations which cannot be reduced to a product of twobody correlations, already introduced at the BHF level. Let us consider in some detail how the three hole-line diagrams can be summed up exactly, in analogy to the summation of the ladder two hole-line diagrams of the BHF approximation. Indeed, since the two hole-line contribution has been summed up by introducing the G-matrix, which is the in-medium two-body scattering matrix, it is therefore conceivable that the three hole-line diagrams could be summed up by introducing some similar generalization of the scattering matrix for three particles. The three-body scattering problem has a long history by itself, and has been given a formal solution by Fadeev [6]. For three distinguishable particles the three-body scattering matrix $T^{(3)}$ is expressed as the sum of three other scattering matrices, $T^{(3)}=T_{1}+T_{2}+T_{3}$. The scattering matrices $T_{i}$ satisfy a system of three coupled integral equations. The kernel of this set of integral equations contains explicitly the two-body scattering matrices pertaining to each possible pair of particles. Also in this case, therefore, the original two-particle interaction disappears from the equations in favour of the two-body scattering matrix. For identical particles the three integral equations reduce to one, because of symmetry. In fact, the three functions $T_{i}$ must coincide within a change of variable with a unique function, which we can still call $T^{(3)}$. The analogous equation and scattering matrix in the case of nuclear matter (or other many-body systems in general) has been introduced by Bethe [7],8]. The integral equation, the Bethe-Fadeev equation, reads schematically

$$
\begin{aligned}
T^{(3)}=G+G X \frac{Q_{3}}{e} & T^{(3)} \\
\left\langle k_{1} k_{2} k_{3}\left|T^{(3)}\right| k_{1}^{\prime} k_{2}^{\prime} k_{3}^{\prime}\right\rangle= & \left\langle k_{1} k_{2}|G| k_{1}^{\prime} k_{2}^{\prime}\right\rangle \delta_{K}\left(k_{3}-k_{3}^{\prime}\right)+ \\
& +\left\langle k_{1} k_{2} k_{3}\left|G_{12} X \frac{Q_{3}}{e} T^{(3)}\right| k_{1}^{\prime} k_{2}^{\prime} k_{3}^{\prime}\right\rangle .
\end{aligned}
$$

The factor $Q_{3} / e$ is the analogous of the similar factor appearing in the integral equation for the two-body scattering matrix $G$, see Eq. (4). Therefore, the projection operator $Q_{3}$ imposes that all the three particle states lie above the Fermi energy, and the denominator $e$ is the appropriate energy denominator, namely the energy of the three-particle intermediate state minus the entry energy $\omega$, in close analogy with the equation for the two-body scattering matrix $G$ of Eq. (4). The real novelty with respect to the two-body case is the operator $X$. This operator interchanges particle 3 with particle 1 and with particle 2, $X=P_{123}+P_{132}$, where $P$ indicates the operation of cyclic permutation of its indices. It gives rise to the so-called "endemic factor" in the Fadeev equations, since it is an unavoidable complication intrinsic to the three-body problem in general. The reason for the appearance of the operator $X$ in this context is that 
no two successive $G$ matrices can be present in the same pair of particle lines, since the $G$ matrix already sums up all the two-body ladder processes. In other words, the $G$ matrices must alternate from one pair of particle lines to another, in all possible ways, as it is indeed apparent from the expansion by iteration of Eq. (7), which is represented in Fig. 7.

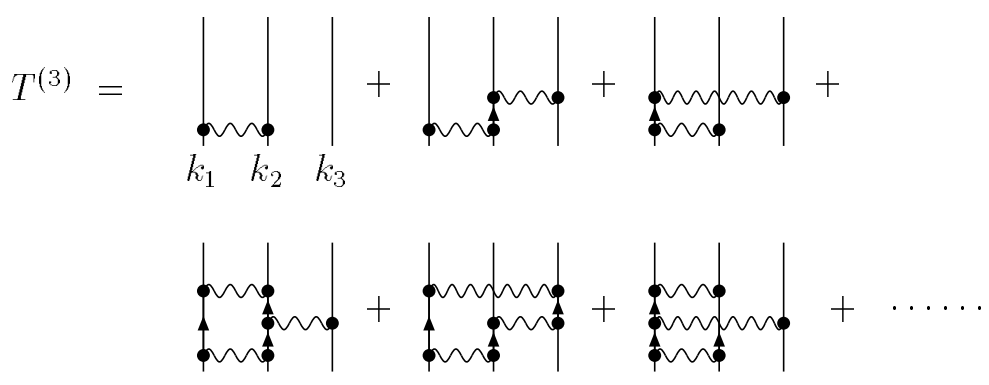

Fig. 7. The first few terms in the expansion of the Bethe-Fadeev integral equation.

Therefore, both cyclic operations are necessary in order to include all possible processes. Adding all terms with an arbitrary number of G-matrices, one gets a generalized ladder series for three-particles, analogous to the ladder series introduced for the two particles case in defining the G-matrix. Indeed, this is the basis for the integral equation (7). In the structure of Eq. (7) the third particle, with initial momentum $k_{3}$, is somehow singled out from the other two. This choice is arbitrary, but it is done in view of the use of the Bethe-Fadeev equation within the BBG expansion.

In order to see how the introduction of the three-body scattering matrix $T^{(3)}$ allows to sum up the three hole-line diagrams, we first notice, following B.D.Day [9], that this set of diagrams can be divided into two distinct groups. The first one includes the graphs where two hole-lines, out of three, originate at the first interaction of the graph and terminate at the last one without any further interaction in between. Schematically the sum of this group of diagram can be represented as in part (a) of Fig. 8.

The third hole-line has been explicitly indicated, out from the rest of the diagram. The remaining part of the diagram describes the rescattering, in all possible way, of three particle-lines, since no further hole-line must be present in the diagram. This part of the diagram is indeed the three-body scattering matrix $T^{(3)}$, and the operator $Q_{3}$ in Eq. (7) assures, as already mentioned, that only particle lines are included.

The second group includes the diagrams where two of the hole-lines enter their second interaction at two different vertices in the diagram, as represented in part (b) of Fig 8. Again the remaining part of the diagram is $T^{(3)}$, i.e. the sum of the amplitudes for all possible rescattering process of three particles. It 
( a )

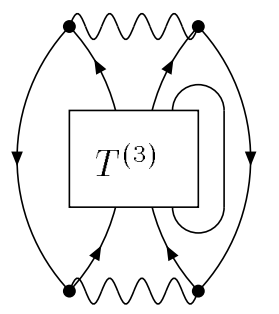

( b )

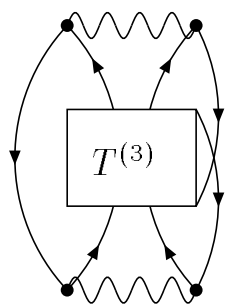

Fig. 8. Schematic representation of the direct (a) and exchange (b) three hole-line diagrams.

is easily seen that no other structure is possible. The set of diagrams indicated in part (b) can be obtained by the ones of part (a) by simply interchanging the final (or initial) point of one of the "undisturbed" hole-line with the final (or initial) point of the third hole-line. This means that one can obtain each graph of the group depicted in Fig. 8b by acting with the operator $X$ on the bottom of the corresponding graph of Fig. Ba. In this sense the diagrams of Fig. \&b can be considered the "exchange" diagrams of the ones in Fig. Ba (not to be confused with the term "exchange" introduced previously for the matrix elements of $G$ ). If one inserts the terms obtained by iterating Eq. (7) inside these diagrams in substitution of the scattering matrix $T^{(3)}$ (the box in Fig. 8), the first diagram, coming from the inhomogeneous term in Eq. (7), is just the bubble diagram of Fig. . The corresponding exchange diagrams is the so called "ring diagram" of Fig. 9 .

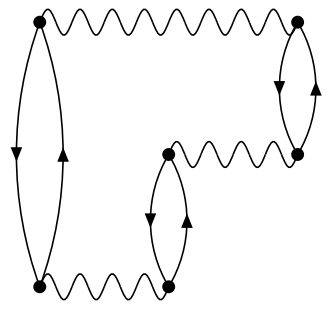

Fig. 9. The ring diagram, belonging to the set of three hole-line diagrams. It can be considered the exchange diagram of the bubble diagram. 
It is easy to draw the remaining series of diagram which one obtains by going on with the iterations.

Once the Bethe-Fadeev equations are solved, the contribution of the direct three hole-line diagrams of Fig. \&a can be written as

$$
\begin{gathered}
E_{3 h}^{d i r}=\frac{1}{2} \sum_{k_{1}, k_{2}, k_{3} \leq k_{F}} \sum_{\left\{k^{\prime}\right\},\left\{k^{\prime \prime}\right\} \geq k_{F}}\left\langle k_{1} k_{2}|G| k_{1}^{\prime} k_{2}^{\prime}\right\rangle_{A} . \\
. \frac{1}{e}\left\langle k_{1}^{\prime} k_{2}^{\prime} k_{3}^{\prime}\left|X T^{(3)} X\right| k_{1}^{\prime \prime} k_{2}^{\prime \prime} k_{3}^{\prime \prime}\right\rangle \frac{1}{e^{\prime}}\left\langle k_{1}^{\prime \prime} k_{2}^{\prime \prime}|G| k_{1} k_{2}\right\rangle_{A}
\end{gathered}
$$

In Eq. (8) the denominator $e=e_{k_{1}^{\prime}}+e_{k_{2}^{\prime}}-e_{k_{1}}-e_{k_{2}}$, and analogously $e^{\prime}=$ $e_{k_{1}^{\prime \prime}}+e_{k_{2}^{\prime \prime}}-e_{k_{1}}-e_{k_{2}}$. The exchange diagrams of Fig. $8 \mathrm{~b}$ can be obtained by multiplying the same expression by a further factor $X$. In summary, the entire set of three hole-line diagrams can be obtained by multiplying the expression of Eq. (8) by $1+X$.

It has been recognized a long ago [8] that the summation of all three-hole diagrams is essential, since individual three-hole diagram can be quite large, but strong cancellation occurs among the different contributions. This is particularly true for the bubble diagram of Fig. 5a and the ring diagram of Fig. 9, which turn out to be quite large but of opposite sign. As already mentioned, the potential insertion diagram of Fig. $5 \mathrm{~b}$ is different from zero in the continuous choice and it turns out to be essential in compensating the contribution of both bubble and ring diagrams. A scheme of approximation was first devised by B.D. Day [9] within the gap choice for the single particle potential. In this scheme the bubble and ring diagrams are indeed singled out from the whole set of three hole-line diagrams, while the remaining series of diagrams is summed up by solving the Bethe-Fadeev integral equation. The bubble diagram requires special numerical treatment, since very large partial waves contribute to the intermediate G-matrix. Once the bubble and ring diagrams are subtracted from the Bethe-Fadeev equation, the resulting integral equation for the whole set of the higher order diagrams turns out to be much less sensitive to the larger partial waves. We will refer to this contribution as the "higher order" contribution. The numerical solution of the Bethe-Fadeev integral equation is delicate. The main difficulty is the large matrix to be inverted to get the scattering matrix $T^{(3)}$. This difficulty can be overcome by introducing a separable representation of the G-matrix appearing in the kernel of the integral equation, as already performed by B.D. Day [9] in the case of the gap choice. We refer to this reference and to ref. [1] for other details of the numerical methods.

The degree of cancellation among the different terms is apparent in Fig. 10, where the bubble, ring and higher order contributions are displayed [10] in the case of the gap choice and the Argonne $\mathrm{v}_{14} \mathrm{NN}$ potential.

The final result, denoted as "total", is relatively small and much smaller in size than the individual contributions. The corresponding results for the continuous choice are displayed in Fig. 11. In this case the additional contribution (BUBU) of the potential insertion diagram in Fig. $5 \mathrm{~b}$ must be considered. One can see the relevance of this term in comparison with the others and its role in determining 


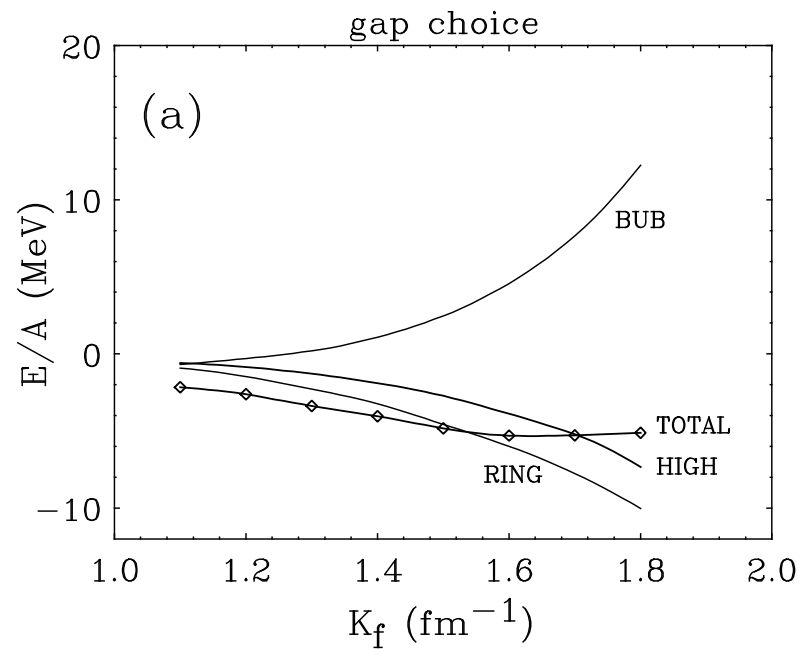

Fig. 10. The contributions of the bubble (BUB), ring (RING) and higher order (HIGH) diagrams to the binding energy of symmetric nuclear matter as a function of Fermi momentum, calculated within the gap choice. The line denoted by TOTAL is the sum of all these contributions and gives the overall three hole-line contribution to the EOS.

the size of the total three hole-line contribution. The latter turns out to be much smaller in the continuous choice than in the gap choice.

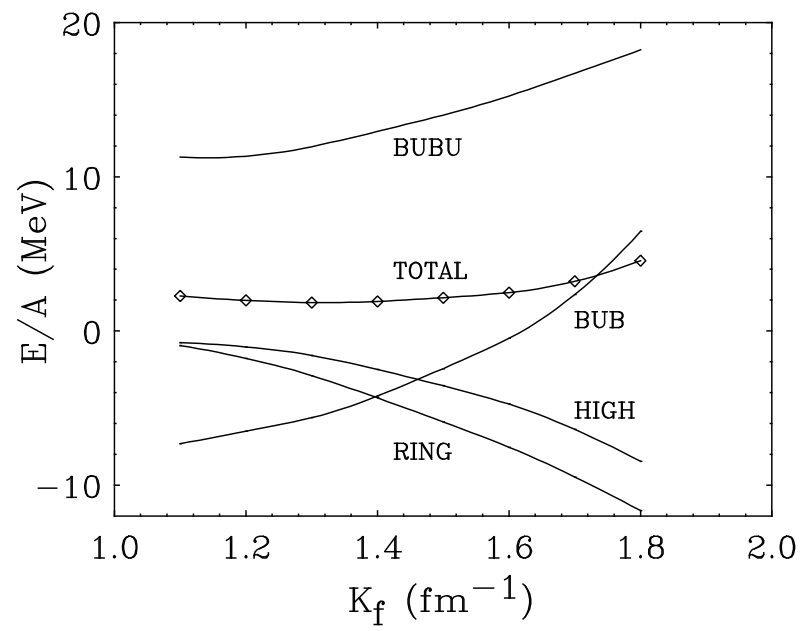

Fig. 11. The same as in Fig. 10, but within the continuous choice. Here the line denoted by BUBU is the contribution of the potential insertion diagram of Fig. Eb. 
The final Equation of State obtained by adding the three hole-line contribution is reported in Fig. 12, both for the gap choice (squares) and the continuous choice (stars), again for the Argonne $\mathrm{v}_{14}$ potential, for a much wider range of densities than in Fig. 6. For comparison, the EOS at the two hole-line level in the continuous choice is also again reported (solid line) from Fig. 6. Two conclusions can be drawn from these results.

i) The two saturation curves in the gap and continuous choices, with the inclusion of the three hole-line diagrams, tend now to collapse in a single EOS, with some deviations only at the highest density. This is a strong indication that a high degree of convergence has been reached at this level of the expansion, according to the criterion discussed above. Notice that the saturation curves extend from a density which is about one half of saturation density to about five times saturation density, and, therefore, it appears unlikely that the agreement between the two choices can be considered as a fortuitous coincidence.

ii) The Brueckner two hole-line EOS within the continuous choice turns out to be already close to the full EOS, since in this case the three hole-line contribution is quite small. In first approximation one can adopt the BHF results with the continuous choice as the nuclear matter EOS.

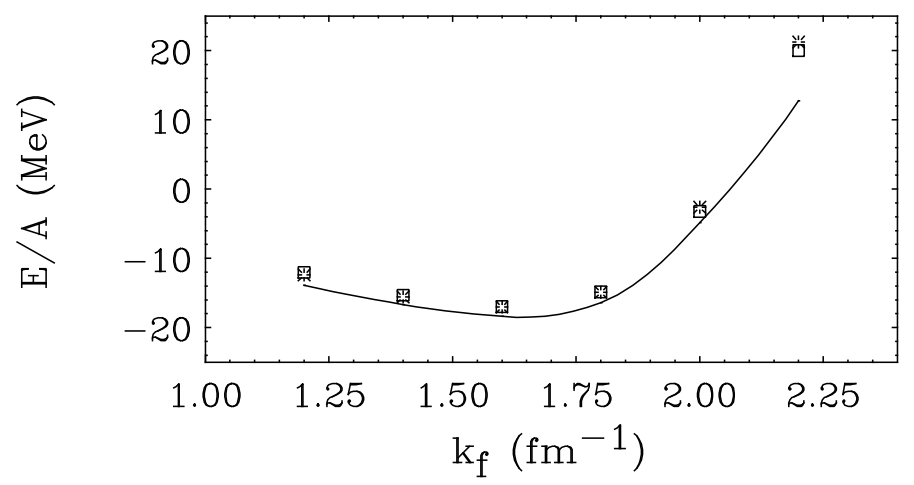

Fig. 12. The Nuclear Equation of State including the three hole-line contribution within the gap choice (squares) and the continuous choice (stars), for the Argonne $\mathrm{v}_{14}$ potential. For comparison, the EOS at the two hole-line level in the continuous choice is also reported (solid line).

The phenomenological saturation point for symmetric nuclear matter is, however, not reproduced, which confirms the finding in ref. [9]. The binding energy per particle at the minimum of the saturation curve turns out to be close to the empirical value of about $-16 \mathrm{MeV}$, but the corresponding density comes out about 30-40 \% larger than the empirical one. Usually this drawback is corrected by introducing three-body forces in the nuclear hamiltonian, and indeed all realistic two-nucleon forces, which fit the experimental two-nucleon phase shifts and deuteron data, are not able to reproduce the empirical saturation point. In 
other words, the results indicate that the missing of the saturation point is not due to a lack of accuracy in the treatment of the nuclear many-body problem, but to a defect of the nuclear hamiltonian. The need of three-body forces in nuclear matter is consistent with the findings in the study of few nucleon systems, where also the binding energy and radii, as well as scattering data, cannot be reproduced with only two-body forces. Not surprisingly, the effects of three-body forces seem to be more pronounced in nuclear matter than in few body systems.

The standard NN interaction models are based on the meson-nucleon field theory, where the nucleon is considered an unstructured point-like particle. The Paris, the Argonne $v_{14}$ (with the improved version $v_{18}$ [1]), and the set of Bonn potentials 12 fall in this category. In the one-boson exchange potential (OBEP) model one further assumes that no meson-meson interaction is present and each meson is exchanged in a different interval of time from the others. However, the nucleon is a structured particle, it is a bound state of three quarks with a gluon-mediated interaction, according to Quantum Chromodynamics (QCD). The absorption and emission of mesons can be accompanied by a modification of the nucleon structure in the intermediate states, even in the case of NN scattering processes, in which only nucleonic degrees of freedom are present asymptotically. A way of describing such processes is to introduce the possibility that the nucleon can be excited ("polarized") to other states or resonances. The latter can be the known resonances observed in meson-nucleon scattering. At low enough energy the dominant resonance is the $\Delta_{33}$, which is the lowest in mass. If the internal nucleon state can be distorted by the presence of another nucleon, the interaction between two nucleons is surely altered by the presence of a third one. This effect produces clearly a definite three-body force, which is absent if the nucleons are considered unstructured. The simplest of such process is depicted in Fig. 13b.
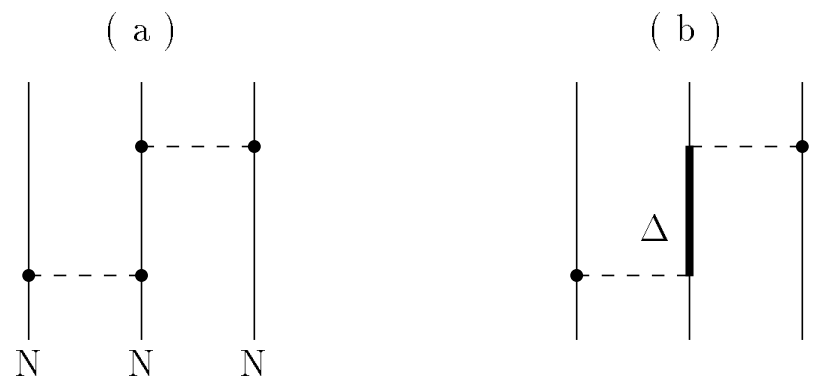

Fig. 13. An interaction process among three nucleons with only two-body force (a), and a process involving a genuine three-body force (b).

Such a process can be interpreted in different but equivalent ways. One way is to view the pion (meson) coming from the first nucleon to polarize the second one, which therefore interacts with a third one as a $\Delta_{33}$ resonance, surely in a different 
way than if it had remained a nucleon, like in Fig. 13a. The process of Fig. $13 \mathrm{a}$ is not indeed a three-nucleon force, but just a repetition of a two-nucleon force. The introduction of a three-nucleon interaction is a consequence of viewing processes like the one of Fig. 13b as an effective interaction among three nucleons, which eventually will be medium-dependent. The genuine three-nucleon forces can be extracted from processes like the one of Fig. 13b by projecting out the $\Delta_{33}$ (or other resonances) degrees of freedom in some approximate way. The theory of three-nucleon forces has a very long history, and it started to be developed since the early stage [13] of the theory of nuclear matter EOS, as well as of few nucleon systems 14. The most extensive study of the three-nucleon forces (TNF) has been pursued by Grangé and collaborators 15. Fig. 14, reproduced from Ref. 16], indicates some of the processes which can give rise to TNF. Graph of Fig. 14a is a generalization of the process of Fig. 13 b, where other nucleon resonances (e.g. the Roper resonance) can appear as intermediate virtual excitation and other exchanged mesons can be present. Graph 14b includes possible non-linear meson-nucleon coupling, as demanded by the chiral symmetry limit 16]. Graph 14c is the simplest one which includes meson-meson interaction. Other processes of this type are of course possible 115, 16], which involves other meson-meson couplings, and they should be included in a complete treatment of TNF. Diagram 14 $\mathrm{d}$ describes the effect of the virtual excitation of

( a )

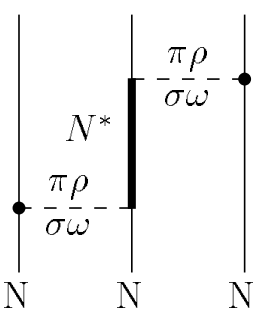

( c )

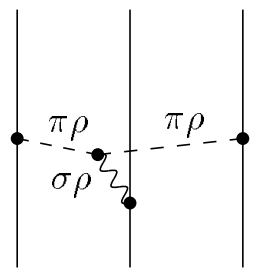

( b )

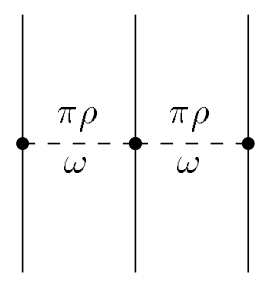

(d)

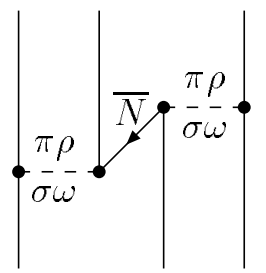

Fig. 14. Some of the processes which can produce a genuine three-body force.

a nucleon-antinucleon pair, and it is therefore somehow of different nature from the others. It gives an important (repulsive) contribution and it has been shown 
[17 to describe the relativistic effect on the EOS to first order in the ratio $\mathrm{U} / \mathrm{m}$ between the single particle potential and the nucleon rest mass.

The $\sigma$ meson, appearing in some of the diagrams, is a hypothetical scalar meson, believed to be responsible for the intermediate attraction in the two-nucleon interaction, whose mass and coupling constant are treated as parameters. One should therefore be careful, as discussed in Ref. [15], to be at least consistent between the treatments of the two-nucleon and the three-nucleon forces. A complete calculations of the TNF in the framework of the meson-nucleon theory, i.e. the calculation of the "best" TNF, is not yet available.

A simpler possibility is to adopt a more phenomenological approach, like the one followed by the Urbana group [18]. Since the EOS obtained with only two-body forces seems to need additional attraction at lower density and an additional repulsion at higher density, it is therefore conceivable that the main effect of TNF can be schematized by one attractive and one repulsive term, as representative of the whole set of three-nucleon processes. Actually, once the usual static approximation is made for the nucleons and the resonances in calculating the meson exchange process, the structure of the different threebody forces turns out to be quite similar. Since the strengths of the different vertex appearing in these diagrams cannot be considered fairly well known, one can treat the strengths of the two representative terms as free parameters to be fitted to some known physical quantities. More explicitly, the TNF is written as

$$
V_{i j k}=V_{i j k}^{2 \pi}+V_{i j k}^{R}
$$

The first (attractive) contribution is a cyclic sum over the nucleon indices $i, j, k$ of products of anticommutator $\{$,$\} and commutator [,] terms$

$$
\begin{aligned}
V_{i j k}^{2 \pi} & =A \sum_{c y c}\left(\left\{X_{i j}, X_{j k}\right\}\left\{\tau_{i} \cdot \tau_{j}, \tau_{j} \cdot \tau_{k}\right\}\right. \\
& \left.+\frac{1}{4}\left[X_{i j}, X_{j k}\right]\left[\tau_{i} \cdot \tau_{j}, \tau_{j} \cdot \tau_{k}\right]\right)
\end{aligned}
$$

where

$$
X_{i j}=Y\left(r_{i j}\right) \sigma_{i} \cdot \sigma_{j}+T\left(r_{i j}\right) S_{i j}
$$

is the one-pion exchange operator, $\sigma$ and $\tau$ are the Pauli spin and isospin operators, and $S_{i j}=3\left[\left(\sigma_{i} \cdot r_{i j}\right)\left(\sigma_{j} \cdot r_{i j}\right)-\sigma_{i} \sigma_{j}\right]$ is the tensor operator. $Y(r)$ and $T(r)$ are the Yukawa and tensor functions, respectively, associated to the one-pion exchange, as in the two-body potential. The repulsive part is taken as

$$
V_{i j k}^{R}=U \sum_{c y c} T^{2}\left(r_{i j}\right) T^{2}\left(r_{j k}\right)
$$

The strengths $A(<0)$ and $U(>0)$ can be fitted to reproduce the ground state energy of both three nucleon systems (triton and ${ }^{3} \mathrm{He}$ ), and the empirical nuclear matter saturation point.

One such a fit, within the Brueckner approximation, is reported in Fig. 15. The empirical saturation point is now reproduced and the EOS become much more repulsive at high density. Of course, the higher density region, needed e.g. 


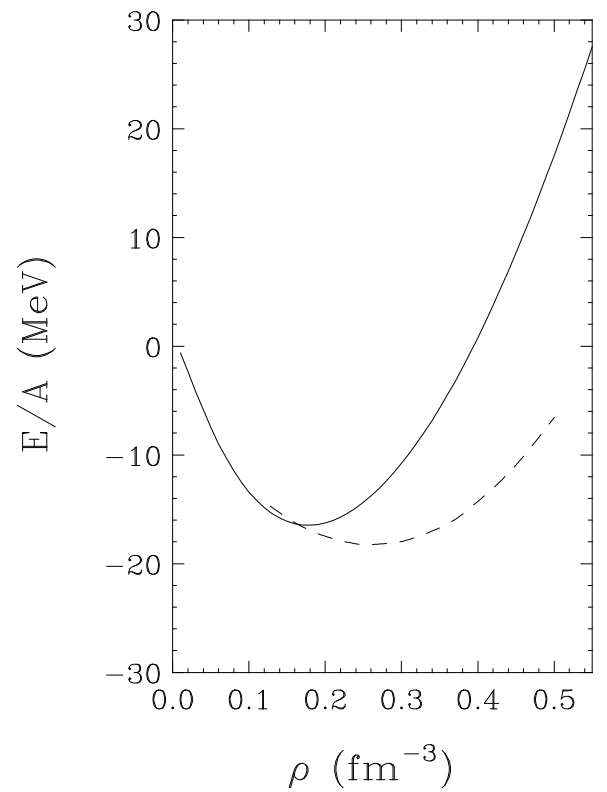

Fig. 15. Saturation curve for symmetric nuclear matter in the Brueckner approximation without (dashed curve) and with (full line) three-body forces.

in neutron star studies, is obtained by extrapolating the TBF from the region around saturation where they are actually adjusted. This EOS can therefore be inaccurate at the higher densities. One can see indeed that the contribution of the three-body forces is substantial at high density, and therefore an accurate inclusion of the three-body forces is highly demanded.

More detail on the use of phenomenological three-body forces will be given in the Section on neutron stars.

\section{The EOS for pure neutron matter}

In this Section we will extend the analysis to pure neutron matter EOS, which is more appropriate for neutron star studies, at densities up to about five times the saturation one. Moreover, we consider the calculations for two nucleon-nucleon potentials, the $\mathrm{Av}_{14}$ and the $\mathrm{Av}_{18}$, in order to analyse the dependence of the results on the nuclear interaction.

We will not give the detail about the contributions of different diagrams, but simply illustrate the results for the neutron EOS, obtained by including only two-body forces. The neutron matter EOS [19] is reported (full lines) in Figs. 16 and 17, both for the continuous choice (BHFC) and standard choice (BHFG). As for symmetric nuclear matter, the discrepancy between the two curves indicates to what extent the EOS still depends on the choice of the auxiliary potential at BHF level, and therefore the degree of convergence. The EOS for the $\mathrm{Av}_{18}$ appears more repulsive, but the trend for the two potentials is similar. The 
discrepancy does not exceed $4 \mathrm{MeV}$ in the whole density range for the $\mathrm{Av}_{14}$ potential, and it is tiny in the case of $\mathrm{Av}_{18}$, except for the highest densities. It is also substantially smaller than in the symmetric nuclear matter case, where the discrepancy is large as much as about $8 \mathrm{MeV}$ at $k_{F}=1.8 \mathrm{fm}^{-1}$ for the $\mathrm{Av}_{14}$ - According to our criterion, this suggests a smaller value of the three hole-line

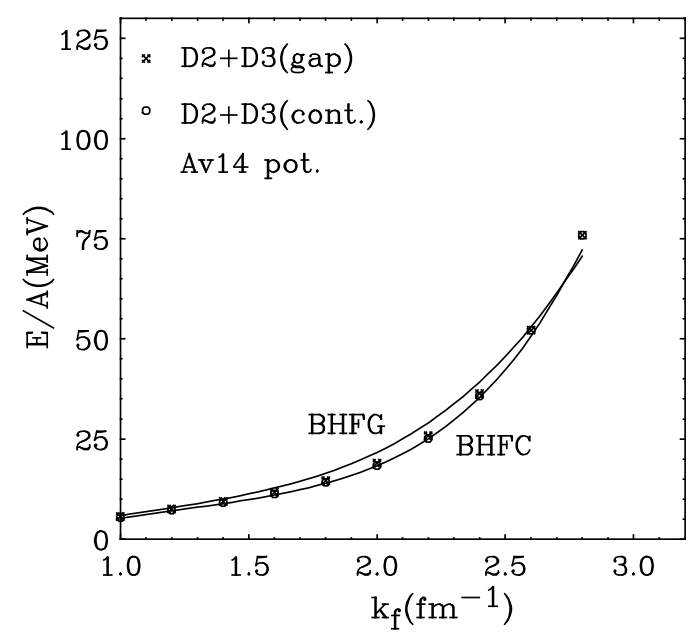

Fig. 16. Equation of state of pure neutron matter for the Av14 nucleon-nucleon potential. The two full lines correspond to the Brueckner-Hartree-Fock approximation, in the gap (BHFG) and continuous choice (BHFC) respectively. The addition of the three-hole contribution $\mathrm{D}_{3}$ gives the total equation of state for the gap (stars) and continuous choice (open circles) respectively.

contribution and is in agreement with the smaller value in neutron matter of the "wound parameter", which is the smallness parameter of the expansion and should give a rough estimate of the ratio between the three hole-line and the two hole-line contributions (in general between two successive order contributions). It can be estimated by the average depletion of the momentum distribution below the Fermi momentum. Indeed, at densities around the saturation value the wound parameter turns out to be close to 0.1 in neutron matter [20] and about 0.25 in symmetric nuclear matter [21].

These expectations are indeed confirmed by the calculations of the three holeline contributions. The inclusion of the three hole-line contributions results in the two final EOS depicted in Figs. 16 and 17, where the points marked by stars and the circles correspond to the standard and continuous choices, respectively. For both NN potentials, the very close agreement between the two EOS is a strong evidence that the expansion has reached convergence. Notice that, at Brueckner (two hole) level, the EOS for the standard and continuous choices cross at some 


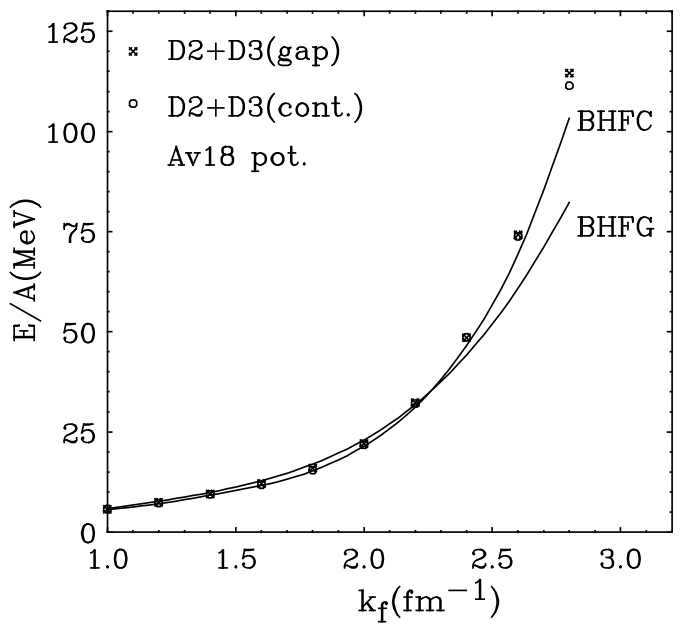

Fig. 17. The same as in Fig. 16, but for the Argonne v18 potential

value of the density, and at that point the overall three hole-line contribution has the same value in both choices. Furthermore, in the continuous choice the three hole-line contribution is substantially smaller, and it is actually negligible to a first approximation. It appears that the corresponding values of the wound parameter, which is close to 0.1 in neutron matter [20], give an upper limit for the ratio between three and two hole-line contributions.

The final EOS appears more repulsive at high density for the $\mathrm{Av}_{18}$ than for the $\mathrm{Av}_{14}$ potential. At lower density, up to about $k_{F}=2.0 \mathrm{fm}^{-1}$, the two potentials produce very close EOS. This is not surprising, since both potentials fit the NN experimental phase shifts up to $350 \mathrm{MeV}$ Lab energy, which indeed corresponds to a relative momentum of about $2.0 \mathrm{fm}^{-1}$. Above this density, the main contribution to the EOS comes from values of the relative NN momentum which need extrapolation beyond the region where the potentials have been fitted to the empirical data. It is likely that different extrapolations are obtained from different potentials in general, and therefore the EOS at high density is largely dependent on the NN potential model, even without the inclusion of three-body forces. The inclusion of three-body forces in pure neutron matter is discussed in the next Section.

\section{Neutron Star Structure}

Once the Nuclear Equation of State, both for symmetric and pure neutron matter, has been established on a firm basis, one can try to study the structure of neutron star (NS) interior. It is indeed believed that the interior of neutron stars is mainly formed by asymmetric nuclear matter with increasing density towards the center. The exterior crust should be actually formed by different state 
of matter at sub-nuclear density, namely crystal structures of atomic species of increasing mass number. In the region where the nuclei start to drip, the crystalline structure is probably mixed with a neutron gas, until nuclei merge into uniform asymmetric nuclear matter. This outer region is the place where many interesting phenomena occur. However, to the extent that the analysis is restricted to the mass and radius of the star, the main contribution is coming from the interior, where nuclear matter sets in. One can hope, therefore, that neutron stars could be a testing ground for the Nuclear Equation of State. The neutron star masses are usually affected by large uncertainties and independent measurements of the radii have not yet been performed. Only recently some indirect indications of neutron star radii have been reported, and, as already noticed, the astrophysics of neutron stars is rapidly developing. An accurate enough measurement of both mass and radius of a neutron star is expected to produce an enormous advancement in our knowledge in the nuclear Equation of State.

The observed neutron star masses are $\approx(1-2) M_{\odot}$ (where $M_{\odot}$ is the mass of the sun, $M_{\odot}=1.99 \times 10^{33} \mathrm{~g}$ ). Typical radii of NS are thought to be of order $10 \mathrm{~km}$, and the central density is a few times normal nuclear matter density $\left(\rho_{0} \approx 0.17 \mathrm{fm}^{-3}\right)$. This requires a detailed knowledge of the EOS for densities $\rho \gg \rho_{0}$. This is a very hard task from the theoretical point of view. In fact, whereas at densities $\rho \approx \rho_{0}$ the matter consists mainly of nucleons and leptons, at higher densities several species of particles may appear due to the fast rise of the baryon chemical potentials with density. Among these new particles are strange baryons, namely, the $\Lambda, \Sigma$ and $\Xi$ hyperons. Due to its negative charge, the $\Sigma^{-}$hyperon is the first strange baryon expected to appear with increasing density in the reaction $n+n \rightarrow p+\Sigma^{-}$, in spite of its substantially larger mass compared to the neutral $\Lambda$ hyperon $\left(M_{\Sigma^{-}}=1197 \mathrm{MeV}, M_{\Lambda}=1116 \mathrm{MeV}\right)$. Other species in stellar matter may appear, like $\Delta$ isobars along with pion and kaon condensations. It is therefore mandatory to generalize the study of nuclear EOS with the inclusion of the possible hadrons, other than nucleons, which can spontaneously appear in the inner part of a NS, just because their appearance is able to lower the ground state energy of the nuclear matter dense phase. In the following we will concentrate on the production of strange baryons and assume that a baryonic description of nuclear matter holds up to densities as those encountered in the core of neutron stars.

As we have seen from the previous Sections, the nuclear EOS can be calculated with good accuracy in the Brueckner two hole-line approximation within the continuous choice for the single particle potential, since the results in this scheme are quite close to the full convergent calculations which include also the three hole-line contribution. It is then natural to include the hyperon degrees of freedom within the same approximation to calculate the nuclear EOS needed to describe the NS interior. To this purpose, one needs also a nucleon-hyperon (NY) and a hyperon-hyperon (YY)interaction. In the following this interaction will be taken as the Nijmegen soft-core model [22]. In the calculations the hyperonhyperon interaction will be neglected in first approximation. We will comment 
on this point in the sequel. With these NN and NY potentials, the various $G$ matrices are evaluated by solving numerically the Brueckner equation, which can be written in operatorial form as

$$
G_{a b}[W]=V_{a b}+\sum_{c} \sum_{p, p^{\prime}} V_{a c}\left|p p^{\prime}\right\rangle \frac{Q_{c}}{W-E_{c}+i \epsilon}\left\langle p p^{\prime}\right| G_{c b}[W],
$$

where the indices $a, b, c$ indicate pairs of baryons and the Pauli operator $Q$ and energy $E$ determine the propagation of intermediate baryon pairs. In a given nucleon-hyperon channels $c=(N Y)$ one has, for example,

$$
E_{(N Y)}=m_{N}+m_{Y}+\frac{k_{N}^{2}}{2 m_{N}}+\frac{k_{Y}^{2}}{2 m_{Y}}+U_{N}\left(k_{N}\right)+U_{Y}\left(k_{Y}\right) .
$$

The hyperon single-particle potentials within the continuous choice are given by

$$
U_{Y}(k)=\operatorname{Re} \sum_{N=n, p} \sum_{k^{\prime}<k_{F}^{(N)}}\left\langle k k^{\prime}\left|G_{(N Y)(N Y)}\left[E_{(N Y)}\left(k, k^{\prime}\right)\right]\right| k k^{\prime}\right\rangle
$$

and similar expressions of the form

$$
U_{N}(k)=\sum_{N^{\prime}=n, p} U_{N}^{\left(N^{\prime}\right)}(k)+\sum_{Y=\Sigma^{-}, \Lambda} U_{N}^{(Y)}(k)
$$

apply to the nucleon single-particle potentials. The nucleons feel therefore direct effects of the other nucleons as well as of the hyperons in the environment, whereas for the hyperons there are only nucleonic contributions, because of the missing hyperon-hyperon potentials. The equations (13 16) define the BHF scheme with the continuous choice of the single-particle energies. Due to the occurrence of $U_{N}$ and $U_{Y}$ in Eq. (14) they constitute a coupled system that has to be solved in a self-consistent manner for several Fermi momenta of the particles involved. Once the different single-particle potentials are known, the total nonrelativistic baryonic energy density, $\epsilon$, and the total binding energy per baryon, $B / A$, can be evaluated

$$
\begin{aligned}
\frac{B}{A} & =\frac{\epsilon}{\rho_{n}+\rho_{p}+\rho_{\Sigma^{-}}+\rho_{\Lambda}} \\
\epsilon & =\sum_{i=n, p, \Sigma^{-}, \Lambda} \int_{0}^{k_{F}^{(i)}} \frac{d k k^{2}}{\pi^{2}}\left(m_{i}+\frac{k^{2}}{2 m_{i}}+\frac{1}{2} U_{i}(k)\right)
\end{aligned}
$$

As we have seen, nonrelativistic calculations, based on purely two-body interactions, fail to reproduce the correct saturation point of symmetric nuclear matter, and three-body forces among nucleons are needed to correct this deficiency. In the sequel the so-called Urbana model will be used, which consists, as we have already seen, of an attractive term due to two-pion exchange with excitation of an intermediate $\Delta$ resonance, and a repulsive phenomenological central 
term. We introduced the same Urbana three-nucleon model within the BHF approach (for more details see Ref. [23]). In our approach the TBF is reduced to a density dependent two-body force by averaging on the position of the third particle, assuming that the probability of having two particles at a given distance is reduced according to the two-body correlation function. The corresponding nucleon matter EOS (no hyperon) satisfies several requirements, namely (i) it reproduces correctly the nuclear matter saturation point, (ii) the incompressibility is compatible with values extracted from phenomenology, (iii) the symmetry energy is compatible with nuclear phenomenology, (iv) the causality condition is always fulfilled.

If leptons, namely electrons and muons, and hyperon are introduced, the general EOS can be calculated for a given composition of the baryon components. This allows the determination of the chemical potentials (by simple numerical derivatives of the energy) of all the species, baryonic and leptonic, which are the fundamental input for the equations of chemical equilibrium. The latter determines the actual detailed composition of the dense matter and therefore the EOS to be used in the interior of neutron stars. Indeed, at high density the matter composition is constrained by three conditions: i) chemical equilibrium among the different species, ii) charge neutrality, and iii) baryon number conservation. At density $\rho \approx \rho_{0}$ the stellar matter is composed of a mixture of neutrons, protons, electrons, and muons in $\beta$-equilibrium [electrons are ultrarelativistic at these densities, $\left.\mu_{e}=\left(3 \pi^{2} \rho x_{e}\right)^{1 / 3}\right]$. In that case the equations for chemical equilibrium read

$$
\begin{aligned}
& \mu_{n}=\mu_{p}+\mu_{e}, \\
& \mu_{e}=\mu_{\mu} .
\end{aligned}
$$

Since we are looking at neutron stars after neutrinos have escaped, we set the neutrino chemical potential equal to zero. Strange baryons appear at density $\rho \approx(2-3) \rho_{0}$ [24], mainly in baryonic processes like $n+n \rightarrow p+\Sigma^{-}$and $n+n \rightarrow n+\Lambda$. The equilibrium conditions for those processes read

$$
\begin{aligned}
& 2 \mu_{n}=\mu_{p}+\mu_{\Sigma}, \\
& \mu_{n}=\mu_{\Lambda} .
\end{aligned}
$$

The other two conditions of charge neutrality and baryon number conservation allow the unique solution of a closed system of equations, yielding the equilibrium fractions of the baryon and lepton species for each fixed baryon density. They read

$$
\begin{aligned}
& \rho_{p}=\rho_{e}+\rho_{\mu}+\rho_{\Sigma}, \\
& \rho=\rho_{n}+\rho_{p}+\rho_{\Sigma}+\rho_{\Lambda} .
\end{aligned}
$$

Finally, from the knowledge of the equilibrium composition one determines the equation of state, i.e., the relation between pressure $\mathrm{P}$ and baryon density $\rho$. It can be easily obtained from the thermodynamical relation

$$
P=-\frac{d E}{d V} .
$$


being $\mathrm{E}$ the total energy and V the total volume. Equation (25) can be explicitly worked out in terms of the baryonic and leptonic binding energies, respectively $B$ and $E_{L}$,

$$
\begin{aligned}
& P=-\frac{d E}{d V}=-\frac{d}{d V}\left(B+E_{L}\right)=P_{B}+P_{L} \\
& P_{B}=\rho^{2} \frac{d(B / A)}{d \rho}=\rho^{2} \frac{d}{d \rho}\left[\left(x_{n}+x_{p}\right) \frac{\epsilon_{N N}}{\rho_{N}}+x_{\Sigma} \frac{\epsilon_{N \Sigma}}{\rho_{\Sigma}}+x_{\Lambda} \frac{\epsilon_{N \Lambda}}{\rho_{\Lambda}}\right] \\
& P_{L}=\rho^{2} \frac{d\left(E_{L} / A\right)}{d \rho}=\rho^{2} \frac{d}{d \rho}\left[x_{e^{-}} \frac{\epsilon_{e^{-}}}{\rho_{e^{-}}}+x_{\mu^{-}} \frac{\epsilon_{\mu^{-}}}{\rho_{\mu^{-}}}\right] .
\end{aligned}
$$

In the above equations $x_{i}$ represent the baryon fraction of each species. As far as the leptons are concerned, at those high densities electrons are a free ultrarelativistic gas, whereas muons are relativistic. Therefore their energy densities $\epsilon_{L}$ are well-known from textbooks, see e.g. ref. 225]. In order to construct models of neutron stars, one needs to calculate the total mass-energy density $\mathcal{E}$ as well. This can be easily obtained just adding the mass-energy densities of each species $\mathcal{E}_{i}$

$$
\mathcal{E}=\mathcal{E}_{N}+\mathcal{E}_{\Sigma}+\mathcal{E}_{\Lambda}+\mathcal{E}_{e^{-}}+\mathcal{E}_{\mu^{-}},
$$

While the electron and muon contributions, respectively $\mathcal{E}_{e^{-}}$and $\mathcal{E}_{\mu^{-}}$, are known from textbooks, the baryonic contribution are given by

$$
\begin{aligned}
& \mathcal{E}_{N}=\frac{1}{c^{2}}\left(\epsilon_{N N}+m_{N} \rho_{N}\right), \\
& \mathcal{E}_{\Sigma}=\frac{1}{c^{2}}\left(\epsilon_{N \Sigma}+m_{\Sigma} \rho_{\Sigma}\right), \\
& \mathcal{E}_{\Lambda}=\frac{1}{c^{2}}\left(\epsilon_{N \Lambda}+m_{\Lambda} \rho_{\Lambda}\right) .
\end{aligned}
$$

being $m_{i}$ the rest mass and $c$ the speed of light. For more details, the reader is referred to ref. [24] and references therein.

In figure 18 we show the chemical composition of $\beta$-stable and asymmetric nuclear matter containing hyperons. In the upper panel we display the case when only two-body nucleonic forces are present, whereas in panel b) nucleonic TBF's are included. We observe that the inclusion of TBF's shifts the hyperon onset points down to $\rho \simeq 2-3$ times normal nuclear matter density, since some additional repulsion is now present. Moreover, an almost equal percentage of nucleons and hyperons are present in the stellar core at high densities. A strong deleptonization of matter takes place, since it is energetically convenient to maintain charge neutrality through hyperon formation than $\beta$-decay. This can have far reaching consequences for the onset of kaon condensation. The main physical features of the nuclear EOS which determine the resulting compositions are essentially the symmetry energy of the nucleon part of the EOS and the hyperon single particle potentials inside nuclear matter. Since at low enough density the nucleon matter is quite asymmetric, the small percentage of protons feel a deep 


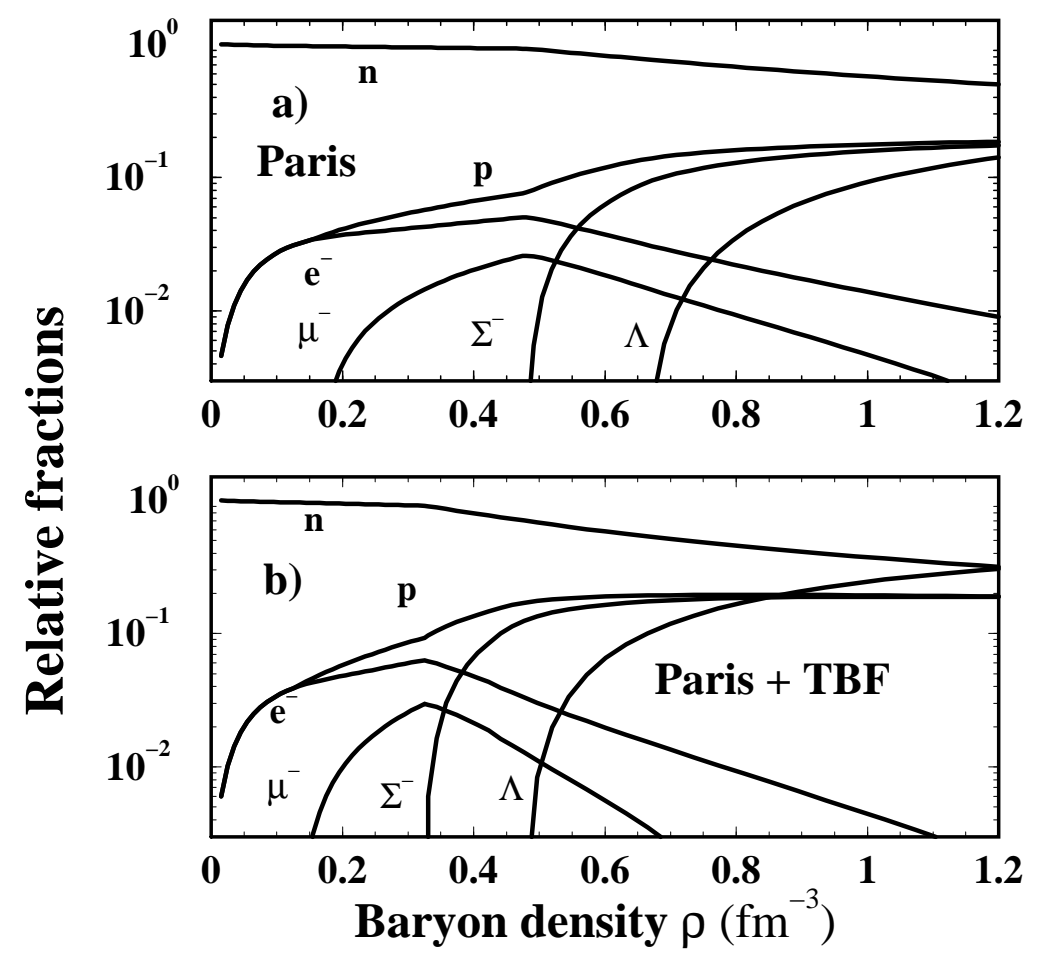

Fig. 18. The equilibrium composition of asymmetric and $\beta$-stable nuclear matter containing $\Sigma^{-}$and $\Lambda$ hyperons is displayed. In the upper panel only two-body nucleonic forces are present, whereas in the lower panel TBF's have been included.

single particle potential, and therefore it is energetically convenient to create a $\Sigma^{-}$hyperon since then a neutron must be converted into a proton. The deepness of the proton potential is mainly determined by the nuclear matter symmetry energy. Furthermore, the potential felt by the hyperons can shift substantially the threshold density at which each hyperon set in. This points are illustrated in Fig. 19, where the different single particle potentials are plotted at a given nucleon density. For simplicity, neutron and proton densities are fixed, given by $\rho_{N}=0.4 \mathrm{fm}^{-3}$ and $\rho_{p} / \rho_{N}=0.1$, and the $\Sigma^{-}$density is varied. Under these conditions the $\Sigma^{-}$single-particle potential is sizably repulsive, while $U_{\Lambda}$ is still attractive (see also Ref. 24]) and the nucleons are much strongly bound. The $\Sigma^{-}$single-particle potential has a particular shape with an effective mass $m^{*} / m$ slightly larger than 1 , whereas the lambda effective mass is typically about 0.8 and the nucleon effective masses are much smaller. 


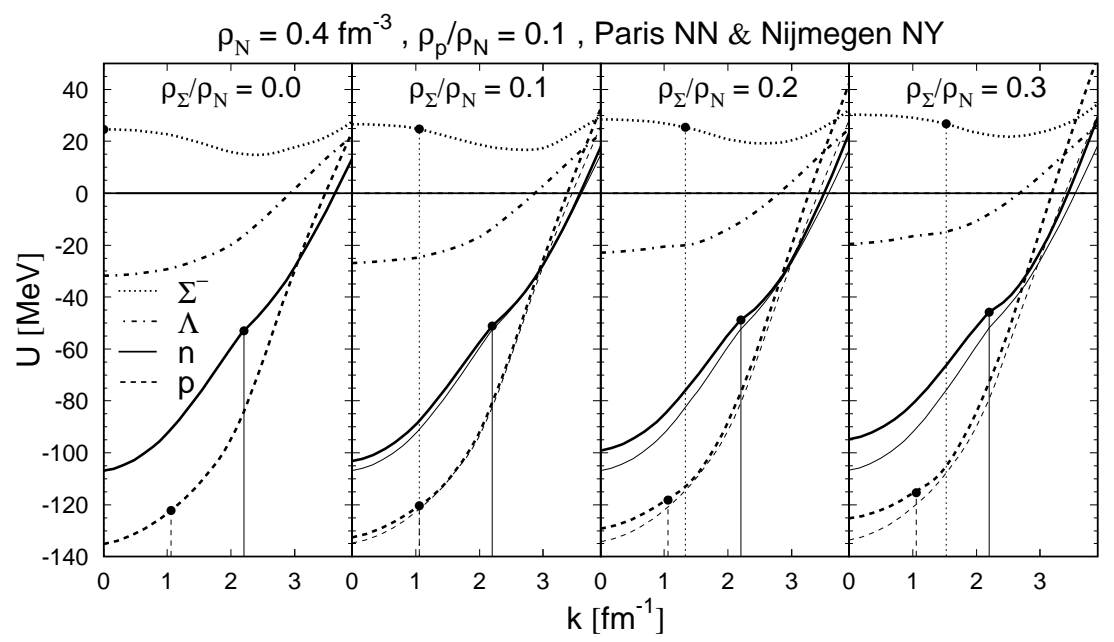

Fig. 19. The single-particle potentials of nucleons $n, p$ and hyperons $\Sigma, \Lambda$ in baryonic matter of fixed nucleonic density $\rho_{N}=0.4 \mathrm{fm}^{-3}$, proton density $\rho_{p} / \rho_{N}=0.1$, and varying $\Sigma$ density $\rho_{\Sigma} / \rho_{N}=0.0,0.1,0.2,0.3$. The vertical lines represent the corresponding Fermi momenta of $n, p$, and $\Sigma$. For the nucleonic curves, the thick lines represent the complete single-particle potentials $U_{N}$, whereas the thin lines show the values excluding the $\Sigma$ contribution, i.e., $U_{N}^{(n)}+U_{N}^{(p)}$.

The resulting Equation of State is displayed in Figure 20. The dotted line represents the case when only two-body forces are present, whereas the solid line shows the case when TBF's are included. The upper curves show the equation of state when stellar matter is composed only by nucleons and leptons. We mainly observe a stiffening of the equation of state because of the repulsive contribution coming from the TBF's. The inclusion of hyperons (lower curves) produces a soft equation of state which turns out to be very similar to the one obtained without TBF's. This is quite astonishing because, in the pure nucleon case, the repulsive character of TBF at high density increases the stiffness of the EOS, thus changing dramatically the equation of state. However, when hyperons are included, the presence of TBF's among nucleons enhances the population of $\Sigma^{-}$ and $\Lambda$ because of the increased nucleon chemical potentials with respect to the case without TBF, thus decreasing the nucleon population. The net result is that the equation of state looks very similar to the case without TBF, but the chemical composition of matter containing hyperons is very different when TBF are included. In the latter case, the hyperon populations are larger than in the case with only two-body forces. This has very important consequences for the structure of the neutron stars. Of course, this scenario could partly change if 
hyperon-hyperon interactions were known or if TBF would be included also for hyperons, but this is beyond our current knowledge of the strong interaction.

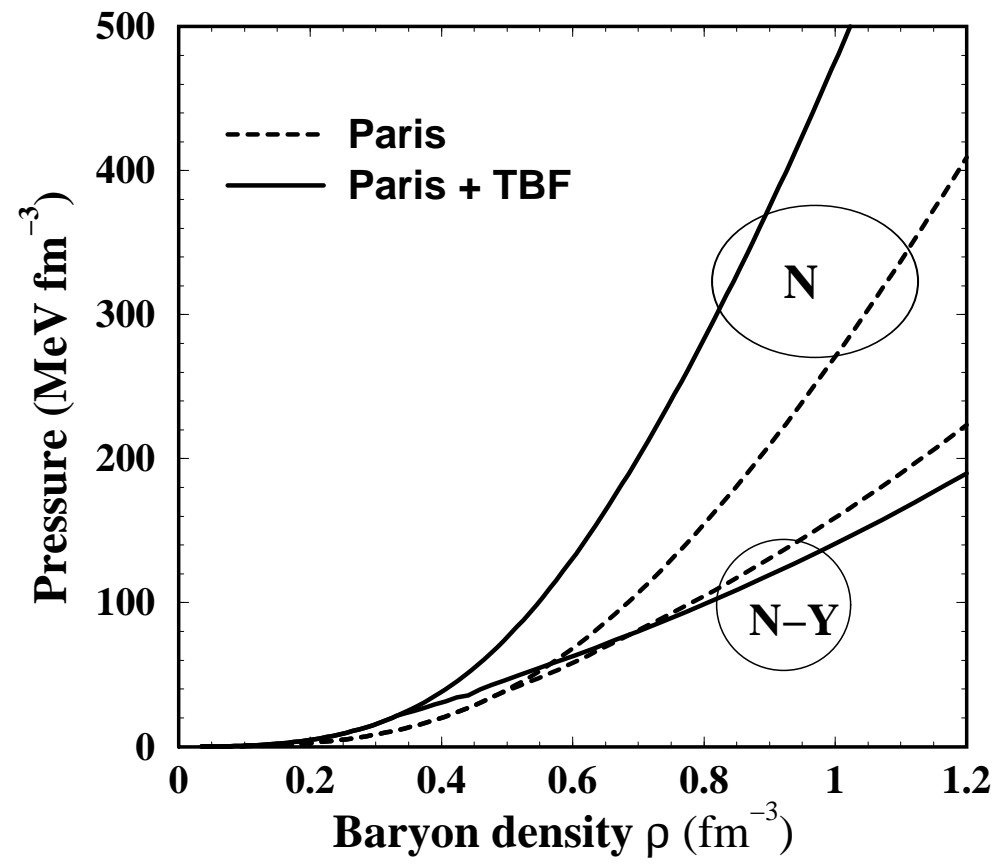

Fig. 20. The pressure is displayed vs. the baryon density for hyperon-free (upper curves) and hyperon-rich (lower curves) matter. The solid (dashed) lines represent the case when nucleonic TBF's are (are not) included.

\section{Equilibrium configurations of neutron stars}

We assume that a star is a spherically symmetric distribution of mass in hydrostatic equilibrium. The equilibrium configurations are obtained by solving the Tolman-Oppenheimer-Volkoff (TOV) equations [25] for the pressure $P$ and the enclosed mass $m$,

$$
\begin{aligned}
\frac{d P(r)}{d r} & =-\frac{G m(r) \mathcal{E}(r)}{r^{2}} \frac{[1+P(r) / \mathcal{E}(r)]\left[1+4 \pi r^{3} P(r) / m(r)\right]}{1-2 G m(r) / r}, \\
\frac{d m(r)}{d r} & =4 \pi r^{2} \mathcal{E}(r),
\end{aligned}
$$

being $G$ the gravitational constant. Starting with a central mass density $\mathcal{E}(r=$ $0) \equiv \mathcal{E}_{c}$, we integrate out until the pressure on the surface equals the one corre- 
sponding to the density of iron. This gives the stellar radius $R$ and the gravitational mass is then

$$
M_{G} \equiv m(R)=4 \pi \int_{0}^{R} d r r^{2} \mathcal{E}(r) .
$$

For the outer part of the neutron star we have used the equations of state by Feynman-Metropolis-Teller [26] and Baym-Pethick-Sutherland [27], and for the medium-density regime we use the results of Negele and Vautherin [28]. For density $\rho>0.08 \mathrm{fm}^{-3}$ we use the microscopic equations of state obtained in the BHF approximation described above. For comparison, we also perform calculations of neutron star structure for the case of asymmetric and $\beta$-stable nucleonic matter. The results are plotted in Fig. 21. We display the gravitational mass $M_{G}$ (in units of the solar mass $M_{o}$ ) as a function of the radius $R$ (panel (a)) and central baryon density $n_{c}$ (panel (b)). We note that the inclusion of hyperons lowers the value of the maximum mass from about $2.1 M_{o}$ down to $1.26 M_{o}$. This
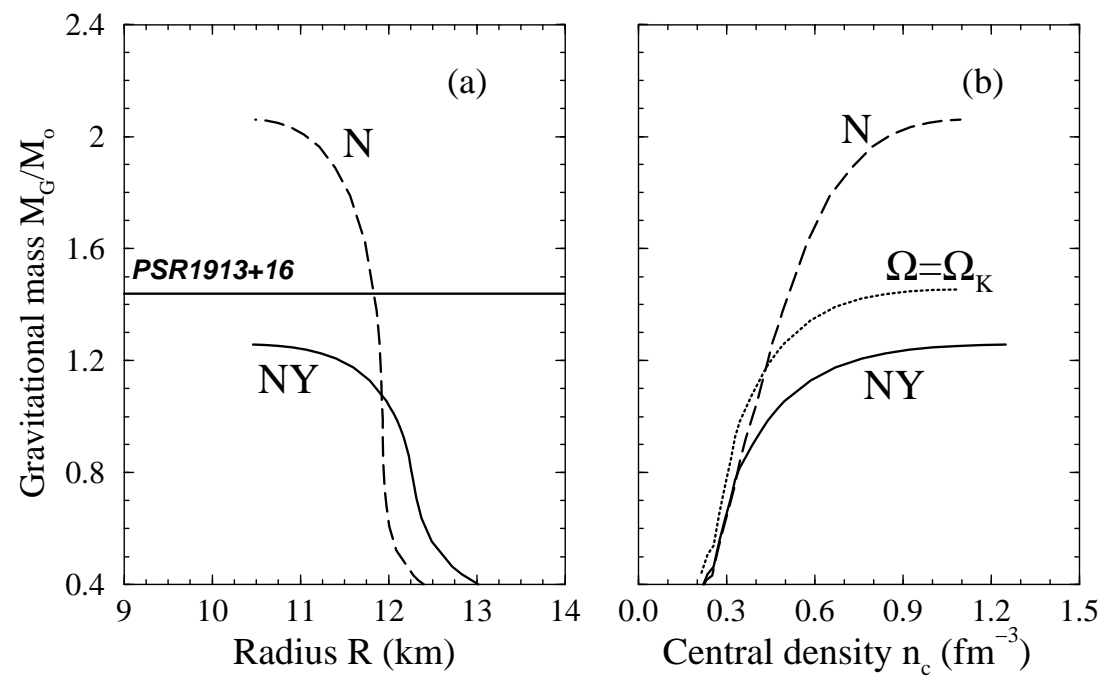

Fig. 21. In panel (a) the mass-radius relation is shown in the case of beta-stable matter with hyperons (solid line) and without hyperons (dashed line). The thick line represents the measured value of the pulsar PSR1913+16 mass. In panel (b) the mass is displayed vs. the central density. The dotted line represents the equilibrium configurations of neutron stars containing nucleons plus hyperons and rotating at the Kepler frequency $\Omega_{K}$.

value lies below the value of the best observed pulsar mass, PSR1916+13, which amounts to 1.44 solar masses. However the observational data can be fitted if rotations are included, see dotted line in panel (b). In this case only equilibrium configurations rotating at the Kepler frequency $\Omega_{K}$ are shown. However, $\Omega_{K}$ is 
much larger than the rotational frequency of that pulsar, and therefore rotation probably does not play any role.

In conclusion, the main finding of our work is the surprisingly low value of the maximum mass of a neutron star, which hardly comprises the observational data. This fact indicates how sensitive the properties of the neutron stars are to the details of the interaction. In particular our result calls for the need of including realistic hyperon-hyperon interactions. However, the use of the available hyperon-hyperon interactions seem to introduce only minor changes in the results [29]. Despite the uncertainty on the NY and YY interactions, it is unlikely that one can obtain a neutron star mass substantially larger. The possible occurrence of a quark core is usually assumed to further soften the EOS and lower the maximum mass. However, this is not necessarily true, since at large density the quark pressure should be close to the one of a ultra-relativistic Fermi gas, which can rise fast enough to stabilize the system. In any case, the possible quark core is not expected to change dramatically the critical neutron star mass. Even if an explicit analysis of the quark core has still to be worked out, it is fair to say that the observation of a neutron star with a mass much larger than 1.4-1.5 solar mass would indicate that indeed some basic ingredient is missing in our understanding of neutron star structure.

\section{Acknowledgments}

The material presented in this contribution is the result of a fruitful collaboration, lasting for several years, with a number of people. Special thanks are due to Dr. I. Bombaci, Prof. L.S. Ferreira, Dr. G. Giansiracusa, Prof. U. Lombardo, Dr. H.-J. Schulze and Prof. H.Q. Song.

\section{References}

1. For a pedagogical introduction, see Nuclear Methods and the Nuclear Equation of State, Edited by M. Baldo, World Scientific, Singapore, International Review of Nuclear Physics Vol. 9, 1999.

2. B.D. Day, Brueckner-Bethe Calculations of Nuclear Matter, Proceedings of the School E. Fermi, Varenna 1981, Course LXXIX, ed. A. Molinari, (Editrice Compositori, Bologna, 1983), p. 1-72; Rev. Mod. Phys. 39, 719 (1967).

3. H.A. Bethe, B.H. Brandow and A.G. Petschek, Phys. Rev. 129, 225 (1962)

4. J. Hüfner and C. Mahaux, Ann. Phys. (N. Y.) 73, 525 (1972).

5. R.B. Wiringa, R.A. Smith and T.L. Ainsworth, Phys. Rev. C29, 1207 (1984).

6. L.D. Fadeev, Mathematical Aspects of the Three-Body Problem in Quantum Scattering Theory, Davey, New York 1965.

7. H.A. Bethe, Phys. Rev. 138, 804 (1965).

8. R.Rajaraman and H.Bethe, Rev. Mod. Phys. 39, 745 (1967).

9. B.D. Day, Phys. Rev. C24, 1203 (1981); Phys. Rev. Lett. 47, 226 (1981);

10. H.Q. Song, M. Baldo, G. Giansiracusa and U. Lombardo, Phys. Rev. Lett. 81, 1584 (1998).

11. R.B. Wiringa, V.G.J. Stocks and R. Schiavilla, Phys. Rev. C51, 38 (1995). 
12. R. Machleidt, Adv. Nucl. Phys. 19, 189 (1989).

13. J. Fuyita and H. Miyazawa, Progr. in Theor. Phys., 17, 360 (1957).

14. Ch. Hadjuk, P.U. Sauer and W. Streuve, Nucl. Phys. A405, 581 (1983).

15. P. Grangé, A. Lejeune, M. Martzolff and J.-F. Mathiot, Phys. Rev C40, 1040 (1989), and references therein.

16. J.-F. Mathiot, Phys. Rep. 173, 63 (1989).

17. G.E. Brown, W. Weise, G. Baym and J. Speth, Comm. Nucl. Part. Phys. 17, 39 (1987).

18. Carlson J., Pandharipande V.R. and Wiringa R.B., Nucl. Phys. A401, 59 (1983).

19. M. Baldo, G. Giansiracusa, U. Lombardo and H. Q. Song, Phys. Lett. B473 (2000) 1.

20. W. Zuo, G.Giansiracusa, U. Lombardo, N. Sandulesco and H.-J. Schulze, Phys. Lett. B421, 1 (1998).

21. W. Zuo, U. Lombardo, and H.-J. Schulze, Phys. Lett. B432, 241 (1998).

22. P. Maessen, Th. Rijken, and J. de Swart, Phys. Rev. C40, 2226 (1989).

23. M. Baldo, I. Bombaci, and G. F. Burgio, Astron. and Astrophys. 328, 274 (1997).

24. M. Baldo, G. F. Burgio, and H.-J. Schulze, Phys. Rev. C 61, 055801-1 (2000).

25. S. L. Shapiro and S. A. Teukolsky, Black Holes, White Dwarfs and Neutron Stars (John Wiley \& Sons, New York, 1983).

26. R. Feynman, F. Metropolis, and E. Teller, Phys. Rev. C 75, 1561 (1949);

27. G. Baym, C. Pethick, and D. Sutherland, Astrophys. Journ. 170, 299 (1971).

28. J. W. Negele and D. Vautherin, Nucl. Phys. A 207, 298 (1973).

29. I. Vidaña, A. Polls, A. Ramos, L. Engvik, and M. Hjorth-Jensen, preprint University of Barcellona, 1999. 